\title{
Presentación del dossier. Socioformación, sostenibilidad y educación
}

Introduction to Dossier.

Socioformation, sustainability and education

María Elena Calles-Santoyor

Universidad Autónoma de Tamaulipas México

Tampico, México

mecalles@docentes.uat.edu.mx

Lourdes Rodríguez-Peralta

Instituto Politécnico Nacional - México

Ciudad de México, México

mlrodriguezp@ipn.mx

\section{La socioformación: un modelo pedagógico para el desarrollo social sostenible}

En el año 2020 surge como innovación pedagógica el enfoque de la socioformación, cuya esencia es retomar el espíritu humanista de la educación, poniendo en primer plano el trabajo colaborativo entre las personas para resolver los problemas comunitarios y del ambiente, con apoyo en las tecnologías de la información y la comunicación, a través del proyecto ético de vida y la vinculación con la sociedad, lo cual implicó dejar atrás el énfasis en el individuo y lo académico, propio de la educación tradicional (Ambrosio, 2018). Este enfoque empuja al talento humano a abordar problemas contextualizados, con base en la colaboración, la flexibilidad, el mejoramiento continuo y la articulación de saberes de diferentes áreas (Martínez et al., 2017; Tobon et al., 2015). mismos estudiantes, sus familias y comunidades.

La socioformación se basa en la sociedad del conocimiento (Krüger, 2006) que consiste en crear y gestionar el conocimiento para afrontar las necesidades con apoyo en la tecnología. Es por ello que el reto de la socioformación es formar ciudadanos que creen y gestionen el conocimiento para resolver los problemas del mundo de la vida, con innovación, emprendimiento y uso de las tecnologías de la información y la comunicación, a través de proyectos transversales que aporten soluciones a los problemas que enfrentan los habitantes de Latinoamérica. Es por ello que Tobón y López (2015) plantean que en esta perspectiva educativa:

Se necesitan docentes en el marco del enfoque socioformativo, que centren a los estudiantes como protagonistas de su propio aprendizaje y los hagan partícipes de la resolución de problemáticas del entorno, de esta manera, podrán comprometerse y al mismo tiempo darán sentido a sus aprendizajes, aplicándolos para una mejora en una meta en común (p. 76).

La socioformación tiene una serie de características que lo hacen diferente de otros modelos pedagógicos. Una primera característica es que promueve el emprendimiento. Este proceso es cada vez más visto como un elemento determinante de los resultados de crecimiento y desarrollo. Vázquez et al., (2006) refieren que los programas de estudio se centran en la formación profesional del estudiante, pero dejan muchos vacíos en encausar los proyectos emprendedores con miras a lo social. Fortalecer la enseñanza universitaria mediante su incorporación al contexto social y laboral, es ofrecer respuestas creativas e innovadoras a los problemas sociales que nos envuelven (Palomares \& Chisvert, 2014). 
Una segunda característica de la socioformación es el énfasis en el proyecto ético de vida. Este concepto es diferente del proyecto de vida propuesto en la psicología y consiste en trabajar con otras personas para mejorar las condiciones de vida en la comunidad y cuidar el ambiente, a la vez que se busca el propio desarrollo personal y se actúa en el entorno a partir de los valores universales como la honestidad, el respeto, la responsabilidad, la solidaridad y la empatía, entre otros. Para Luna-Nemecio (2020) es la realización plena del ser humano dentro de la sociedad, contemplando y acatando las normas universales que fomentan el orden, el respeto, y la honestidad. Por ende, se trata de un eje profundamente social y comprometido con el desarrollo sostenible.

Una tercera característica de la socioformación son las habilidades de pensamiento complejo. Al respecto, en la socioformación se hace un replanteamiento del pensamiento complejo tal y como lo propuso Morin, y se orienta en torno a habilidades para afrontar los retos del entorno, tales como la resolución de problemas, el pensamiento crítico, la metacognición, el análisis sistémico y la creatividad (Tobón \& Luna-Nemecio, 2021). Estas habilidades están articuladas entre sí y ayudan a que el sistema educativo pueda implementar acciones concretas para que se desarrollen en las personas, a través de buscar el desarrollo sostenible. Además, cada habilidad en sí misma ha tenido una mejora en su conceptualización, como es la metacognición. Esta tradicionalmente ha consistido en un proceso de toma de consciencia y autorregulación con cuatro elementos: persona, tarea, estrategia y entorno (Izquierdo-Merlo, 2020). En la socioformación se convierte en una autoevaluación personal y en equipo que se práctica de manera continua para mejorar el desempeño acorde con los valores universales (Tobón, 2013).

Una cuarta característica de la socioformación es la cocreación y la gestión del conocimiento. Esto trasciende el énfasis de la sociedad de la información de buscar, procesar y aplicar la información. Gimeno $(2010,2013)$ expone que lo prioritario de los sistemas educativos es la calidad de los conocimientos y su adquisición por parte de los estudiantes. En la socioformación, más que buscar y procesar información, lo que se busca es cocrear el conocimiento con base en la colaboración para diagnosticar las necesidades, comprenderlas, explicarlas y ejecutar soluciones eficaces y prácticas en los problemas contextuales, de tal manera que haya continuidad en las acciones implementadas.

Una quinta característica de la socioformación es la inclusión. Este proceso se encuentra presente en la agenda política, educativa y social de muchas organizaciones de Latinoamérica. Al respecto, Vásquez et.al (2017) refieren que la inclusión respeta la condición física, cognitiva o sociocultural de las personas. Sin embargo, este concepto tiende a enfocarse en buscar que la comunidad incluya a las personas con discapacidad, con lo cual es un proceso unidireccional. En la socioformación, la inclusión es bidireccional y se basa en buscar que todos los individuos trabajen en manera colaborativa en proyectos para resolver problemas aportando sus recursos, habilidades y saberes a través de la complementariedad de acciones.

\section{El desarrollo social sostenible desde la socioformación}

La pandemia ocasionada por el COVID-19 evidenció aún más diversos vacíos, riesgos y vulnerabilidades (Luna-Nemecio, 2020); la poca responsabilidad social de los ciudadanos para afrontar la infección; la falta de resiliencia para adaptarse a los cambios en lo personal, familiar, social y laboral; y la contaminación ambiental tanto en espacios rurales, pero, sobre todo, urbanos (Luna-Nemecio, 2021). Por ello, es esencial la transformación de las ciudades en ciudades sostenibles (Luna-Nemecio et al., 2020). En este sentido, el desarrollo social sostenible desde la socioformación busca lograr los objetivos del milenio, como son: la eliminación de la pobreza y el hambre; la mejora en los servicios de salud y bienestar; tener un servicio educativo pertinente; lograr impacto en el cuidado del ambiente para disminuir el calentamiento global a través de acciones e intervenciones en la 
parte social, cultural, científica, y tecnológica, en armonía con el contexto ambiental local y global, actual y futuro, a través de la formación integral de las personas y comunidades con apoyo en la tecnología y la implementación de proyectos transversales (Martínez-Iñiguez et al., 2021).

Así, el desarrollo social sostenible desde la socioformación toma como base el componente social, económico y ambiental, se caracteriza por enfocarse en la formación integral del ser humano, bajo distintos escenarios. En cada uno promueve el desarrollo del talento humano de todos los actores de la comunidad (por ejemplo: estudiantes, personal de apoyo a la educación, funcionarios, padres de familia y comunidad); se promueve la resolución de problemas del contexto a través del trabajo colaborativo entre ciudadanos, comunidades y gobiernos; y se busca una cultura ambiental basada en aplicar la innovación para prevenir el calentamiento global y asegurar la biodiversidad (MartínezIñiguez et al., 2021).

\section{La sociedad del conocimiento: una perspectiva humanista y compleja}

El conocimiento, más que la información, es el factor de desarrollo más importante en nuestro tiempo, no se puede circunscribir solamente a los círculos académicos, debe formar parte de las agendas de gobiernos nacionales, regionales, locales y urbanos, de las organizaciones no gubernamentales, de los encargados de la salud, de los empresarios, de los gremios productores y de los ambientalistas (Pescador-Vargas, 2014). La sociedad del conocimiento, de acuerdo con Tobon et al. (2015a, 2015b) propende para que los ciudadanos aprendan a buscar, sistematizar, crear, innovar y aplicar el conocimiento en el marco del trabajo en red para resolver las necesidades del entorno, con lo cual este concepto se articula plenamente al desarrollo social sostenible. De esta forma, hay una importante diferencia con la sociedad de la información, que solamente se centra en procesar la información para realizar tareas muy concretas, sin una visión del desarrollo social.

\section{Currículo socioformativo y desarrollo social sostenible}

Las instituciones educativas han venido implementando el currículo por competencias, con la idea de que están innovando para formar personas líderes y comprometidas. Sin embargo, el currículo por competencias, aunque fue relevante en un momento, actualmente es insuficiente para lograr la formación de ciudadanos comprometidos con el desarrollo social sostenible, porque dicho enfoque está centrado más en actividades y tareas de una determinada área, y considera poco la visión del desarrollo social y el cuidado del ambiente con base en la aplicación de los valores universales en la resolución de los problemas.

En el ámbito educativo, una de las necesidades más acuciantes de los países de Latinoamérica es diseñar procesos curriculares que ayuden en la formación del talento humano que aporte soluciones puntuales a las demandas de las comunidades. Esto requiere concebir qué tipo de personas necesita el continente, qué tipo de personas requiere cada país y cada región específica (Escribano, 2017). Es por ello necesario buscar otras alternativas para implementar el currículo, como es el caso de la socioformación que tiene como propósito aplicar el pensamiento complejo en el entorno con responsabilidad, creatividad y ética (Montes de Oca \& Machado, 2014).

Desde la óptica de la socioformación, el currículo se caracteriza por un conjunto de proyectos formativos interdisciplinarios orientados a abordar retos del contexto que tengan impacto en hacer posible el desarrollo social sostenible. Algunas de las características del currículo socioformativo son: 1) se busca la resolución de problemas del entorno y con base en ello se promueve la formación, como un medio, no como un fin en sí misma (Tobón et al., 2015b); 2) involucra diversos actores, como los docentes, estudiantes, directivos, familia, comunidad y organizaciones (Martínez, 2017); 
3) se trabaja en la mejora continua, por lo que no es indispensable tener un currículo completo para iniciar la formación, sino ante todo construir una visión compartida fuerte, y en camino se van completando los procesos de acuerdo con las necesidades de los actores implicados (Tobón 2007, 2013a, 2013b); y 4) busca mejorar las condiciones de vida y cuidar el ambiente, más que lograr el aprendizaje de contenidos (Tobón, 2014).

\section{Los proyectos formativos: microcurrículo y didáctica}

Los proyectos formativos son una de las principales estrategias propuestas por la socioformación con el fin de formar los ciudadanos que se requieren para lograr el desarrollo social sostenible en Latinoamérica. Además, constituyen una opción factible para estructurar el currículo, con el fin de trascender las asignaturas, materias o módulos que dificultan el abordaje de los retos del mundo real en su complejidad. Un proyecto formativo consiste en un conjunto articulado de actividades para resolver un problema del entorno y contribuir de esta forma a mejorar las condiciones de vida y cuidar el ambiente, buscando un producto relevante. En esta metodología se aborda el trabajo colaborativo, el desarrollo de las habilidades de pensamiento complejo, el proyecto ético de vida, el proceso de emprendimiento y la cocreación del conocimiento enfocado en las necesidades del entorno.

\section{Prácticas pedagógicas para lograr el desarrollo social sostenible}

El currículo socioformativo se implementa a través de prácticas pedagógicas que abordan tanto los docentes como los estudiantes en los diversos espacios de formación: hogar, comunidad, institución educativa, aula, mundos virtuales, etc. Estas prácticas se orientan al abordaje de diferentes retos, con base en los propósitos de formación establecidos en función del aporte al desarrollo social sostenible que se haya planteado. De esta forma, se trasciende el concepto de "competencias docentes", más centradas en tareas de tipo pedagógico que ejecutan los maestros y que son unidireccionales (del maestro hacia el estudiante), por prácticas enfocadas en retos que hacen que los estudiantes tengan más motivación y participación (Arreola et al., 2019; Tobón et al., 2021). Algunas de estas prácticas pedagógicas son: sensibilización de los estudiantes con los retos del entorno, análisis y resolución de retos del contexto, trabajo en el proyecto ético de vida, desarrollo y aplicación de las habilidades de pensamiento complejo, trabajo colaborativo, evaluación socioformativa, inclusión basada en la colaboración, etc.

\section{Artículos que componen el dossier.}

El presente Dossier "Socioformación, sostenibilidad y educación" fue el resultado de una convocatoria a nivel de Latinoamérica por correo y redes sociales con el fin de reunir a diferentes investigadores en torno a la búsqueda de opciones desde la educación para contribuir al desarrollo social sostenible, considerando avances e innovaciones en el área, con una visión inter y transdisciplinaria. Los trabajos tenían que ser fruto de procesos investigativos y proponer nuevas opciones para la formación de acuerdo con las características de la región. Cada artículo recibido fue evaluado por dos investigadores externos a la Revista y a doble ciego. Al respecto, entre las muchas contribuciones recibidas, fueron seleccionados cinco trabajos que se presentan a continuación.

El primer trabajo de investigación es "La sustentabilidad como asignatura pendiente: evaluación de confiabilidad de una guía de observación para la práctica docente con enfoque socioformativo" de los autores Dany Vazquez Ayala y Josemanuel Luna-Nemecio. El propósito de este estudio fue elaborar una guía para observar y analizar la práctica de los docentes desde el enfoque socioformativo, y hacer una validación de su contenido a partir de expertos. De esta manera, las 
instituciones y universidades tendrán un instrumento con el cual diagnosticar las prácticas de los docentes y brindar procesos de acompañamiento con el fin de mejorarlas, de tal manera que los estudiantes desarrollen el talento necesario para afrontar los retos del desarrollo social sostenible. El instrumento aborda cuatro ejes: los contenidos que se trabajan con los estudiantes, las actividades de aprendizaje, la interacción y los recursos para el aprendizaje. Algunos aspectos innovadores de este estudio son: 1) se consideran los retos del contexto; 2) se propende por la colaboración e interacción entre los estudiantes; 3) se considera la organización de los estudiantes en función del tipo de actividades a llevar a cabo; y 4) se apoya a los alumnos en el uso de los recursos. Sin duda, este estudio ofrece una guía para mejorar la práctica docente en diversos niveles educativos y de esta manera ayuda a la implementación de la socioformación.

El segundo trabajo es de la investigadora Faridy Bermeo y se titula "Aplicación de los proyectos socioformativos dentro del aula: experiencia docente en la asignatura de Estadística Descriptiva". Este es un interesante estudio que se hizo con el propósito de presentar una experiencia concreta de aplicación de los proyectos formativos en la educación superior, con estudiantes de ingeniería en el Instituto Politécnico Nacional de México, una de las universidades públicas más grandes y de mayor calidad del país. Para ello se presenta la metodología seguida y los resultados alcanzados con los alumnos. Al respecto, los resultados encontrados muestran que los proyectos formativos tuvieron impacto en un aprendizaje más pertinente de los estudiantes, de acuerdo con los retos de la sociedad del conocimiento, que se basa en crear y gestionar el conocimiento para afrontar las necesidades del entorno. Además, la metodología permitió una mejor vinculación entre los saberes para el abordaje de retos reales en el mundo de la vida. La innovación de este estudio fue la aplicación de los proyectos formativos, una estrategia por esencia socioformativa, en las Matemáticas a nivel de la educación superior, área en la cual se requieren más estudios de este tipo.

El tercer trabajo se titula "Diseño y validez de la escala autoevaluación de las competencias del docente de matemáticas" a cargo del investigador Jesús Antonio Larios-Trejo. El propósito de esta investigación fue diseñar y validar una escala con el fin de que los docentes de Matemáticas autoevalúen sus competencias docentes. Para ello, la escala valora 11 dimensiones tomando en cuenta la socioformación, como por ejemplo: 1) Organiza y anima situaciones de aprendizaje; 2) Gestionar la progresión de los aprendizajes; 3) Elaborar y hacer evolucionar dispositivos de diferenciación; 4) Implicar a los alumnos en sus aprendizajes y en su trabajo; 5) trabajo en equipo; etc. El estudio muestra que la escala tiene adecuados niveles de validez de contenido respecto a la pertinencia y comprensión de las preguntas, por lo cual puede emplearse por parte de los maestros para revisar y mejorar continuamente su práctica pedagógica. La principal innovación de este estudio es el énfasis en la autoevaluación, pues con frecuencia los instrumentos en esta área se elaboran es para ser empleados por las autoridades o estudiantes.

El cuarto trabajo es del investigador René Pérez y se titula "El software como apoyo didáctico en la enseñanza de la electrónica digital en la educación superior en México”. Su propósito es presentar una serie de elementos a tener en cuenta para aplicar el software en la materia de electrónica en el marco de la pandemia por COVID-19, considerando las tendencias en el área. Para ello, se hizo un análisis documental con el fin de documentar los contenidos de la materia, se buscaron los softwares más empleados en la enseñanza de la asignatura y se analizó la adecuación del software a los temas de la materia en el Instituto Tecnológico Nacional de México.

El último artículo que se presenta en este Dossier es "Aprendizajes clave en el marco del Nuevo Modelo Educativo Mexicano" que tiene como autor al investigador Florentino López. El propósito de este estudio fue realizar un análisis documental para lograr una comprensión del concepto de “aprendizajes clave" en el marco de la educación básica y media superior de México, a partir de la 
última reforma educativa que hizo este país en el 2017. Junto a ello, se propone la socioformación como un modelo pedagógico pertinente para lograr los aprendizajes clave en los estudiantes, considerando los retos de la sociedad del conocimiento y del desarrollo social sostenible. Un resultado importante del estudio es que llega al planteamiento de que los aprendizajes clave son los saberes fundamentales que los estudiantes deben aprehender en la educación tomando en cuenta los aprendizajes esperados que están en el plan de estudios, con el fin de que sean competentes en el entorno.

\section{Referencias}

Alba, A. de. (2020). La investigación educativa a través del espejo. Perfiles educativos, 42(169), 3-6. https:// doi.org/10.22201/iisue.24486167e.2020.169.60016

Ambrosio, P. R.(2018). La socioformación: un enfoque de cambio educativo. Revista iberoamericana de educación, 76(1), 57-82. https://doi.org/10.35362/rie7612955

Arreola, A., Palmares, G. y Ávila, G. (2019). La práctica pedagógica desde la socioformación. RAES. 11(18), 74-87. http://www.revistaraes.net/revistas/raes18_art5.pdf

Báscolo, E., Houghton, N., \& Del Riego, A. (2018). Lógicas de transformación de los sistemas de salud en América Latina y resultados en acceso y cobertura de salud. Revista Panamericana de Salud Pública, 42, e126. https://doi.org/10.26633/RPSP.2018.126

De Sousa Santos, B. (2020). La cruel pedagogía del virus. Buenos Aires: CLACSO

Escribano, H, E. (2017). La educación en América Latina: desarrollo y perspectivas. Actualidades Investigativas en Educación ,17(2), 355-377. https://dx.doi.org/10.15517/aie.v1711.28147

Espín, A., y Juanes, B. (2021). Competencias pedagógicas en los docentes de Administración de Empresas de la Universidad Metropolitana de Ecuador. Mendive. Revista de Educación. 19(1), 183-198. http:// scielo.sld.cu/scielo.php?script=sci_arttext\&pid=S1815-76962021000100183\&lng=es\&tlng=es.

Gimeno, J. (comp.) (2010). Saberes e incertidumbres sobre el currículum. Morata.

Gimeno, J. (2013). En busca del sentido de la educación. Morata.

Izquierdo-Merlo, M. E. (2020). Gestión del conocimiento desde el enfoque socioformativo hacia el desarrollo social sostenible. Ecocience International Journal, 2(2), 10-18. https://doi.org/10.35766/je20222

Krüger, K. (2006). El concepto de sociedad del conocimiento. Revista bibliográfica de geografía y ciencias sociales, 11(683), 1-17 https://revistes.ub.edu/index.php/b3w/article/view/25676

Luna-Nemecio, J. (2020). Determinaciones socioambientales del COVID-19 y vulnerabilidad económica, espacial y sanitario-institucional. Revista de Ciencias Sociales, 26(2), 21-26. http://dx.doi.org/10.31876/ rCs.v26i2.32419

Luna-Nemecio, J. (2020). Para pensar el desarrollo social sostenible: múltiples enfoques, un mismo objetivo. Kresearch/ Religación. Centro de Investigaciones en Ciencias Sociales y Humanidades desde América Latina. https://doi.org/10.35766/dss20

Luna-Nemecio, J. (2021). Sustentabilidad y resiliencia: avatares y alternativas para las ciudades frente a la devastación socioambiental en el siglo XXI. Bitácora Urbano Territorial, 31(II): 7-13. https://doi. org/10.15446/bitacora.v31n2.94199

Luna-Nemecio, J., y Tobón, S. (2021). Urbanización sustentable y resiliente ante el Covid-19: nuevos horizontes para la investigación de las ciudades. Revista Universidad y Sociedad. 13(1), 110-118. http:// scielo.sld.cu/pdf/rus/v13n1/2218-3620-rus-13-01-110.pdf

Luna-Nemecio, J., Tobón, S., \& Juárez-Hernández, T. (2020). Sustainability-based on socioformation and complex thought or sustainable social development. Resources, Environment and Sustainability, 2, 10007. https://doi.org/10.1016/j.resenv.2020.100007

Martínez, J.E. (2017). Calidad del currículo en educación superior desde un enfoque socioformativo. En M. d. Gallegos, E. Gallegos, G. Paz y D. G. Toledo, Redes académicas, Docencia e Investigación Educativa (pp. 23-31). REDEM. 
Martínez-Iñiguez, J., Tobón, S. y Soto-Curiel, J. (2021). Ejes claves del modelo educativo socioformativo para la formación universitaria en el marco de la transformación hacia el desarrollo sostenible. Formación Universitaria. 14(1), 53-66. http://dx.doi.org/10.4067/S0718-50062021000100053

Montes de Oca, N., \& Machado, F. (2014). Formación y desarrollo de competencias en educación superior cubana. Revista Humanidades Médicas, 14(1). http://scielo.sld.cu/scielo.php?script=sci_arttext\&pi$\mathrm{d}=\mathrm{S} 1727-$

Palomares, M. D., \& Chisvert, M. J. (2014). Ética y empresa en el espacio universitario: el emprendimiento social en las universidades públicas como vehículo facilitador de la equidad social. REDU: Revista de Docencia Universitaria, 12(2), 205.

Parra- Acosta, H., Tobón, S. \& López- Loya, J. (2015). Docencia socioformativa y desempeño académico en la educación superior. Paradigma, XXXVI(1), 42 - 55.

Pescador-Vargas, B. (2014). ¿Hacia una sociedad del conocimiento? Rev.fac.med, Bogotá , v. 22, n. 2, p. 6-7, https://www.redalyc.org/pdf/910/91039150001.pdf

Rodrigo-Cano, D., Pcó, M. y Dimuro, G. (2019). Los objetivos de Desarrollo Sostenible como marco para la acción y la intervensión social y ambiental. Retos Revista de Ciencias de la Administración y Economía. 9(17), 25-36. https://doi.org/10.17163/ret.n17.2019.02

Sandín, M. (2003). Investigación cualitativa en educación. España. Mc Graw Hill.

Schwab, K. (2016). The fourth industrial revolution. World Economic Forum

SEP. (2017). Modelo educativo 2017. Implicaciones para la reforma en marcha. Temas estratégicos 43. https:// cutt.ly/2mMYdFV

Tobón, S. (2017a, noviembre). Educación pertinente para el desarrollo sustentable en la sociedad del conocimiento. En J. Velez-Ramos (Moderador), The 1st International Conference on Tecnology, Science and Education for Sustainable Development, CITED. Congreso conducido por CORCIEM y el Centro Universitario CIFE, Cuernavaca, México. Descarga de: https://cife.edu.mx/recursos/2018/10/24/icongreso-internacional-de-tecnologia-ciencia-y-educacion-para-el-desarrollosostenible-cited-2017/

Tobón, S. (2017b). Evaluación socioformación. Estrategias e instrumentos. Mount Dora (USA): Kresearch

Tobón, S. (2015). Necesidad de un nuevo modelo educativo para Latinoamérica. Paradigma, 36(2), 5-6. https://doi.org/10.37618/PARADIGMA.1011-2251.2015.p5-6.id558

Tobón, S. (2014). Proyectos formativos: teoría y práctica. Pearson.Ruiz

Tobón, S. (2013a). Formación integral y competencias: pensamiento complejo, currículo, didáctica y evaluación (4ta. ed.). Ecoe Ediciones.

Tobón, S. (2013b). Metodología de gestión curricular: una perspectiva socioformativa. Trillas.

Tobón, S. (2007). El enfoque complejo de las competencias y el diseño curricular por ciclos propedéuticos. Acción Pedagógica, 16(1), 14-28. http://www.saber.ula.ve/bitstream/123456789/17292/2/articulo2.pdf

Tobón, S., González, L., Nambo, J. S. y Vázquez, J. M. (2015a). La socioformación: un estudio conceptual. Paradigma, 36(1), 7-29. http://revistas.upel.edu.ve/index.php/paradigma/article/view/2661/1273

Tobón, S., Guzmán, C. E., Hernández, J. S. y Cardona, S. (2015b). Sociedad del Conocimiento: estudio documental desde una perspectiva humanista y compleja. Paradigma, 36(2), 7-36. https://bit.ly/2MI6loi

Tobón, S., Juárez-Hernández, L., Herrera-Meza, S. y Núñez, C. (2021). Pedagogical Practices: Design and Validation of SOCME-10 Rubric in Teachers who have Recently Entered Basic Education. Psicología Educativa, Revista de los Psicólogos de la Educación. 27(2) 155-165. https://journals.copmadrid.org/ psed/art/psed2021a13

Tobón, S., \& Luna-Nemecio, J. (2021). Complex Thinking and Sustainable Social Development: Validity and Reliability of the COMPLEX-21 Scale. Sustainability, 13(12), 6591. http://doi.org/10.339o/su13126591

Vásquez, J., Hernández, J., Vázquez, J., Juárez, L. \& Guzmán, C. (2017). El trabajo colaborativo y la socioformación: un camino hacia el conocimiento complejo. Revista Educación y Humanismo, 19(33), 334356. http://dx.doi.org/10.17081/eduhum.19.33.2648 